\title{
Effect of ionizing radiation on the bacterial and fungal endophytes of the halophytic plant Kalidium schrenkianum
}

\author{
Jing Zhu ${ }^{1}$, (Xiang) (Sun) ${ }^{2}$, Zhidong Zhang ${ }^{1}$, Qiyong Tang ${ }^{1}$, Meiying Gu ${ }^{1}$, Lijuan Zhang ${ }^{1}$, \\ Min Hou ${ }^{1}$, Amir Sharon ${ }^{2}$, and Hongli Yuan ${ }^{1}$ \\ ${ }^{1}$ Affiliation not available \\ ${ }^{2}$ Tel Aviv University
}

November 20, 2020

\begin{abstract}
Endophytes are microbes found within tissues of plants in various types of terrestrial and aquatic ecosystems, including those habitats with ionizing radiation. Our study investigates the differences in composition of bacterial and fungal endophytes associated with the halophytic plant Kalidium schrenkianum and the effects of geochemical factors and radiation (at low, medium, high level and control) on the community structure of endophytic bacteria and fungi. The bacterial class Actinobacteria and the fungal class Dothideomycetes predominated the endophytic communities of K. schrenkianum. Aboveground parts had higher fungal diversity while belowground parts had higher bacterial diversity. Soil pH, total nitrogen, and organic matter showed significant effects on the diversity of root endophytes. Radiation had no significant effect on the abundance of different bacterial classes. Sordariomycetes predominated the root fungal microbiota under high radiation intensity. Differences in the endophytic communities between aboveground and belowground parts were more than that between the radiation levels. No significant differences were found in the aboveground bacterial communities among the radiation levels. Radiation showed a significant effect on the fungal co-occurrence networks. Negative correlations were found between endophytic bacteria and fungi in the plant. The genetic diversity of both endophytic bacteria and fungi was higher in radioactive environments. Our findings suggest that the endophytes associated with aboveground and belowground parts of K. schrenkianum follow different mechanisms for community assembly and different paradigms in stress response.
\end{abstract}

\section{Introduction}

Endophytes are symbiotic microbes that live within a plant and are important components of the plant microbiome (Porras-Alfaro \& Bayman, 2011). These microbes are found distributed in terrestrial and marine plants and have high phylogenetic diversity and ecological function. Endophytes and their host plants have coevolved, and both benefit from this mutual symbiosis. Studies have shown that endophytes improves host plant physiology to adapt to stressed environments; endophytes reprogrammed the host response to pathogen invasion and increased toxic chemical production to provide protection against herbivores (Arnold et al., 2003; Gange, Eschen, Wearn, Thawer, \& Sutton, 2012; Rodriguez et al., 2008). In the other side, endophytes live inside plant tissues, which protect them from dehydration, poor nutrition, ultraviolet radiation, and competition (Saikkonen, Faeth, Helander, \& Sullivan, 1998; Yao et al., 2020). The mutual interaction profoundly shaped community and changed the biodiversity of plant and microbes, however, the related knowledge remains limited. Therefore, the biology and ecology of endophytic microbiota and their hosts have gained attention and become important research topics.

Endophytic microbial community structure is influenced by various biotic and abiotic factors, such as host identity, climate, biological or circumstantial stresses, and geochemical factors (U'Ren, Lutzoni, Miadlikowska, Laetsch, \& Arnold, 2012). Host identity is the major factor that determines endophytic community 
composition (X. Sun, Ding, Hyde, \& Guo, 2012; U'Ren et al., 2012), whereas environmental factors have a complex influence on the endophytic community. Biotic and abiotic stress alters structure of endophytic communities. Studies have shown that endophytic population is affected by climate changes such as increased carbon dioxide emission, global warming, and drought (Compant, Heijden, \& Sessitsch, 2010). Rodriguez et al. (2008) demonstrated habitat-specific, symbiotically-conferred stress tolerance in plants under high-stress environment. Therefore, research on how endophytic community shift under stressed conditions will shed light into fundamental issues in how the mutual interaction between plants and endophytes promotes the adaption of the symbiotic entity

Ionizing radiation is a type of radiation, in the form of electromagnetic waves or particles, with sufficient energy to ionize an atom or a molecule. Overdosed exposure to ionizing radiation causes harmful effects in living organisms. Ionizing radiation is generally detected in areas exposed to radioactive minerals, in high altitude environments, or in aerospace. Nuclear power plants, nuclear weapon tests, nuclear accidents, and mining produced new radioactive habitats. However, certain plants, animals, and microbes survive under radioactive environment. Studies have reported the existence of shrubs, rodents, terrestrial algae, and fungi in Nevada Test Site (NTS) few years after the nuclear tests (Allred \& Beck, 1963; Beatley, 1964; Rickard \& Beatley, 1965; Shields \& Drouet, 1962; Shields, Durrell, \& Sparrow, 1961). Durrell and Shields (1960) isolated 41 fungal taxa from soil within a mile radius of ground zero sites two years after nuclear tests in NTS. Thirty-seven culturable fungal species of 19 genera were detected on the walls and other building structures in the inner parts of the shelter of the damaged fourth unit of the Chernobyl Nuclear Power Plant (Zhdanova, Zakharchenko, Vember, \& Nakonechnaya, 2000). Radiation has also resulted in shifts in the local microbial communities. For example, soil fungal community structure appeared to shift toward species that may be more radiation resistant, and melanin-containing fungi ascended to dominate the soil fungal communities with increase in radionuclide pollution (Zhdanova, Zakharchenko, \& Haselwandter, 2005). Lavrinienko, Tukalenko, Mappes, and Watts (2018) correlated the gut microbiomes of bank vole Myodes glareolus inhabiting Chernobyl zone with the radioactivity level. Wehrden et al. observed physiological and morphological changes in the organisms of radioactive environments and other negative effects of radiation on the ecosystem (Wehrden et al., 2012). The biological and ecological traits of ecosystem under radiation stress is a great concern in policy making on nuclear power utilization and in designing aerospace sustainable life support system. However, there are no studies on the symbiotic microbes of plants exposed to ionizing radiation.

Endophytic microbes symbiotically associate with host plants, and the symbiotic entity of inter-kingdom jointly challenge harsh circumstances. However, the relationship between endophytes and the host plant under radioactive environment is unknown. High-radiation habitats because of Caesium- $137\left({ }^{137} \mathrm{Cs}\right)$ accumulation from historic nuclear test exist in arid, saline semi-arid desert of northwest China. This paper investigates the endophytic microbes (fungi and bacteria) in the roots and aerial parts of Amaranthaceae halophyte Kalidium schrenkianum (Pall.) Moq., a dominant population of local flora. Here, we collected plant samples and soil samples from sites representing different levels of radiations. We aim to understand the diversity and community structure of endophytic bacteria and fungi in a radioactive habitat; determine the main environmental factor (geochemical characteristics of soil and local radiation level) shaping the endophytic communities; and elucidate the shift in pattern of endophytic microbes with radiation level.

\section{Materials and methods}

\subsection{Study site and sampling}

This study was conducted in Hoxud County in the Xinjiang Uyghur Autonomous Region of China $\left(91^{\circ} 45^{\prime} 42^{\prime \prime} \mathrm{E}, 40 \mathrm{deg} 39^{\prime} 75^{\prime \prime} \mathrm{N}\right)$. This region experiences a semi-arid climate with a mean annual temperature of $12.56 \operatorname{deg} \mathrm{C}$ and a mean annual precipitation of $591 \mathrm{~mm}$. The sampling sites are located in the watershed area of seasonal floods from radiation-contaminated region with ${ }^{137} \mathrm{Cs}$ accumulation. The soil had a salt content above $2 \%$ in the surface layer $(<20 \mathrm{~cm}$ deep) and a radionuclide level $3-5$ fold that of normal soil. K. schrenkianum was the dominant population of the local habitat. Plant materials and soil samples were collected from four sites with different environmental radioactivity levels during August and September 2017 
(Table S1). Five plants (15 m apart) were uprooted from each site. Whole plants were placed in large autoclaved paper envelopes, labelled, transported to laboratory in an icebox, and stored at $-80 \operatorname{degC}$ until further process. Five soil samples were collected with shovels from the surface soil $(0-20 \mathrm{~cm}$ deep) of each site. Soil samples from each site were sieved to remove rocks and plant litter, pooled, packed and labelled in cloth bags, and stored at $4 \operatorname{deg} \mathrm{C}$ to transport to the laboratory. The geochemical characteristics, such as $\mathrm{pH}$, electrical conductivity (EC), organic matter $(\mathrm{OM})$, total nitrogen $(\mathrm{TN})$, soluble nitrogen (SN), available phosphorus $(\mathrm{P})$, available potassium $(\mathrm{K})$, salt (Sal), chloride ion $(\mathrm{Cl})$, sulfate (Sulf), calcium ion $(\mathrm{Ca})$, magnesium ion $(\mathrm{Mg})$, and sodium ion $(\mathrm{Na})$, were measured in the Institute of Quality Standards \& Testing Technology for Agro-Products, Xinjiang Academy of Agricultural Sciences.

\subsection{DNA extraction, PCR, and NGS sequencing}

$K$. schrenkianum plants were divided in to aerial parts and roots to analyze the endophytic microbes, and altogether 40 samples ( 5 individual $\times 4$ treatments $\times 2$ tissue types) were used for DNA extraction. Each plant material (20 g) was surface sterilized with $75 \%$ ethanol for $1 \mathrm{~min}, 3.25 \%$ sodium hypochlorite for $3 \mathrm{~min}$, and $75 \%$ ethanol for $30 \mathrm{~s}$ (Guo, Hyde, \& Liew, 2000). Genomic DNA was extracted following CTAB method (Guo et al., 2000). One gram of the surface-sterilized plant material was freeze-dried using liquid nitrogen, homogenized with a mortar and pestle, transferred to a tube with $5 \mathrm{~mL} 2 \mathrm{x}$ cetyltrimethylammonium bromide (CTAB) extraction buffer (2\% (w/v) CTAB, $100 \mathrm{mM}$ Tris-HCl, $1.4 \mathrm{M} \mathrm{NaCl}, 20 \mathrm{mM}$ EDTA, 1.5\% polyvinylpyrolidone (PVP), $0.5 \%$ 2-mercaptoethanol; $\mathrm{pH} 8.0$; preheated to $65 \mathrm{degC}$ ). The concentration of DNA was measured using a NanoDrop 1000 Spectrophotometer (Thermo Scientific, Wilmington, USA).

The V5-V7 hypervariable region of the bacterial $16 \mathrm{~S}$ ribosomal RNA gene was amplified using $799 \mathrm{~F}$ (AACMGGATTAGATACCCKG) (Chelius \& Triplett, 2001) and 1193R (ACGTCATCCCCACCTTCC) primers (Bodenhausen, Horton, \& Bergelson, 2013). The fungal internal transcribed spacer region 1 (ITS1 region) of ribosomal RNA was amplified using ITS1F (CTTGGTCATTTAGAGGAAGTAA) (Gardes \& Bruns, 1993) and ITS2 (GCTGCGTTCTTCATCGATGC) primers (White, Bruns, Lee, \& Taylor, 1990).

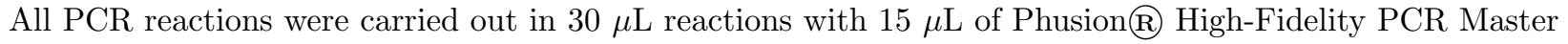
Mix (New England Biolabs, Ipswich, MA); $0.2 \mu \mathrm{M}$ of forward and reverse primers, and about $10 \mathrm{ng}$ template DNA. Thermal cycling consisted of initial denaturation at 98 for $1 \mathrm{~min}, 30$ cycles of denaturation at 98 for $10 \mathrm{~s}$, annealing at 50 for $30 \mathrm{~s}$, and elongation at 72 for $30 \mathrm{~s}$, followed by a final extension at 72 for 5 min. Mix same volume of $1 \times$ loading buffer (contained SYB green) with PCR products and operate electrophoresis on $2 \%$ agarose gel for detection. Then, PCR products was purified with GeneJETTM Gel Extraction Kit (Thermo Scientific, Waltham, MA). Sequencing libraries were generated using Ion Plus Fragment Library Kit 48 rxns (Thermo Scientific, Waltham, MA) following manufacturer's recommendations. The library quality was assessed on the Qubit@ 2.0 Fluorometer (Thermo Scientific, Waltham, MA). At last, the library was sequenced on an Ion S5TM XL platform.

\subsection{Bioinformatic analysis}

Raw reads from bacterial and fungal dataset were demultiplexed and quality filtered using QIIME software (Version1.7.0). Reads with a quality score $<20$ and those lacking complete barcode and primers were excluded from further analysis. Chimeric sequences were removed using USEARCH software. Subsequently, DADA2 workflow (Callahan, Sankaran, Fukuyama, McMurdie, \& Holmesa, 2016) was used to remove singletons and doubletons. Both bacterial and fungal datasets were dereplicated to generate the amplicon sequence variants (ASVs).

Taxonomy was assigned to bacterial and fungal ASVs using Naïve Bayes approach with minimum 75 bootstrap calls following DADA2 workflow (Callahan et al., 2016) against SILVA version 132 (Quast et al., 2013) and UNITE general FASTA release for Fungi version 8.0 (Nilsson et al., 2019), respectively. For bacterial dataset, those ASVs that were not assigned to bacterial genus, were clustered into different operational taxonomic units (OTUs) based on 97\% similarity with function otuin "kmer" package (Wilkinson, 2018). One random sequence was selected from each OTU, and assigned based on SILVA references following the above method. Then the taxonomy assignments of bacterial ASVs and OTUs were combined to an overall 
bacterial taxon-sample table. All ASVs and OTUs that were assigned to non-bacteria, Cyanobacteria phylum, or Rickettsiales order were removed from the overall taxon-sample table. The ASVs or OTUs in the overall table were then agglomerated at the bacterial genus level with identical assignments using "phyloseq" package as described in DADA2 workflow (Callahan et al., 2016). For fungal dataset, those ASVs that were not assigned to fungal species, were similarly clustered, identified based on UNITE references for eukaryotes version 8.0 (Nilsson et al., 2019), generated fungal taxon-sample dataset, filtered off non-fungal taxa, and agglomerated fungal taxa at species level with DADA2 workflow mentioned above. Please see supplementary file 1 and Fig. S1 for more details and schematic diagram.

The ASV and OTU sequences have been deposited in GenBank of National Center for Biotechnology Information under the accession numbers KEBK00000000 (bacterial 16S sequences) and MT351182-MT353643 (fungal ITS1 sequences) and the raw sequences in the Sequence Read Archive of NCBI under BioProject PRJNA625640 (for bacterial data) and PRJNA613597 (for fungal data).

\subsection{Data analysis}

Read counts from all samples were of the same order of magnitude (18,834 to 44,787 for bacterial dataset and 9726 to 62,613 for fungal dataset). Singletons, and doubletons were first filtered out from both bacterial and fungal datasets. To decrease the noise, taxa with the sum of relative abundance less than 0.001 were removed. This resulted in a core dataset of 693 taxa by 40 samples in bacterial dataset and 364 taxa by 40 samples in fungal dataset. The raw taxa counts were normalized to abundance using Hellinger transformation.

Statistical analyses were performed using $\mathrm{R}$ version 3.6.1 (R Development Core Team, 2016). Graphs were plotted with R packages "ggplot2" (Wickham, 2016), "grid" (Murrell, 2005), and "gridExtra" (Auguie, 2017). Two-way analysis of variance (two-way ANOVA) was carried out to test the effect of plant tissue type or radiation level on the richness and diversity of bacterial and fungal microbiota with functionaov in "stats" package (R Development Core Team, 2016). Type 1 error rates had a Benjamini-Hochberg (FDR) $p$ value correction performed for ANOVA models with function p.adjust in "stats" package (Benjamini \& Hochberg, 1995; R Development Core Team, 2016; Veach et al., 2019). Significant differences between the microbial populations were further compared using Tukey's honestly significant difference (HSD) test with function HSD.test in "agricolae" package (Mendiburu, 2019).

The distance matrices of community composition (Hellinger-transformed OTU read data) of endophytic fungi were constructed by calculating dissimilarities using Bray-Curtis method (Faith, Minchin, \& Belbin, 1987). Non-metric multidimensional scaling (NMDS) was used to visualize the community composition dissimilarity of endophytic bacteria or fungi among the different plant tissues or radiation levels usingmetaMDSfunction in "vegan" package (Oksanen et al., 2016). Analysis of similarities (ANOSIM) was applied to statistically test the significant differences in microbial composition between plant tissues or among radiation levels. Permutational multivariate analysis of variance (PerMANOVA) with 999 permutations was implemented with adonis in "vegan" package to investigate the environmental influence on microbiota composition.

The effect of different environmental factors (explanatory variables) on endophyte abundance or richness (genus level for bacteria and species level for fungi) was tested using Poisson generalized linear models (GLM) with stepwise selection by AIC. This analysis was performed using function $g l m$ in "stats" package and function stepAIC in "MASS" package (R Development Core Team, 2016; Veach et al., 2019; Venables \& Ripley, 2002). The data distribution was tested with function shapiro.test in "stats" package. All data were calculated with Poisson distribution and overdispersion in data was tested with function qcc.overdispersion.test in "qcc" package (Scrucca, 2004). Type 1 error rates were FDR-corrected with the method mentioned above.

Co-occurrence analysis was applied on bacterial and fungal datasets separately and collectively with function cor.test in "stats" package (R Development Core Team, 2016). The co-occurrence networks were visualized with "igraph" package (Csardi \& Nepusz, 2006). Network characteristics were determined using functions in "bipartite" package (Dormann, Fründ, Blüthgen, \& Gruber, 2009).

Intra-genus genetic diversity of bacteria and fungi from control and three treatment levels were evaluated by 
computing the pairwise distances of DNA sequences within the groups. Only the ASVs assigned taxonomy at the genus level for bacteria and fungi were included in the analysis. Pairwise distance was calculated among all ASVs available in a certain genus from one treatment level using "K80" model withdist.dna in "ape" package (Paradis \& Schliep, 2018). One-way ANOVA was applied to test the difference significance of intra-genus genetic diversity, as well as all sequence distances regardless of genera, among four treatments.

\section{Results}

\subsection{General description}

The Ion platform produced approximately 2,790,479 raw reads for prokaryotes from 40 samples by sequencing V5-V7 hypervariable region of the bacterial 16S ribosomal RNA gene. After quality control, denoising, and removal of chimera sequences, 1,235,434 high-quality sequences were obtained. A total of 19,367 ASVs recovered in the final dataset were subjected to taxonomy assignment; 8788 ASVs were assigned to different genera. Meanwhile, 10,579 ASVs, which could not be identified at the genus level, were clustered into 2002 OTUs at $97 \%$ identity level and were again subjected to taxonomy assignment. The identified ASVs and OTUs were subsequently combined, and taxa assigned to non-Bacteria, Cyanobacteria, Chloroplast, and Rickettsiales were removed from the dataset. The bacterial dataset agglomerated at the genus level yielded a new dataset covering 1927 taxa across 40 samples with singletons and doubletons removed.

Similarly, 3,232,676 raw reads for eukaryotes were obtained from 40 samples by sequencing ITS1 region of fungal ribosomal RNA. After quality control, denosing, and removal of chimera sequences, 1,704,072 highquality sequences and 14,801 ASVs were obtained. Out of this, 1747 ASVs were assigned to different fungal species. Meanwhile, 13,054 ASVs, which could not be identified at the species level, were clustered into 719 OTUs at $97 \%$ identity level and were again subjected to taxonomy assignment. The identified ASVs and OTUs were subsequently combined, and non-Fungi and plant taxa were removed from the dataset. The dataset agglomerated at the species level yielded a new dataset covering 843 taxa across 40 samples with singletons and doubletons removed.

\subsection{Shifting taxon composition at high hierarchy}

Endophytic fungi showed shifting community composition among treatments at high taxonomical hierarchy. Class Actinobacteria (mostly Actinomycetales) followed by classes Alphaproteobacteria (mostly Rhizobiales and Sphingomonadales) and Gammaproteobacteria (mostly Xanthomonadales and Oceanospirillales) dominated the endophytic bacterial communities in aerial tissues, roots, and the whole plant of $K$. schrenkianum (Fig. 1a). No bacterial class showed significant difference in abundance with radiation level.

Dothideomycetes, Sordariomycetes, and unassigned fungi were the most prevalent fungal groups. Dothideomycetes was the most abundant class in the endophytic fungal communities associated with K. schrenkianum(Fig. 1b). Dothideomycetes significantly predominated the aerial tissues regardless of the radiation level while it was the second most abundant fungal class in the roots (Table S2). Order Pleosporales dominated Dothideomycetes class. Unassigned fungi were the most abundant group in the roots of plants from control, low, and medium radioactive environments while Sordariomycetes (mostly Hypocreales, Microascales, Sordariales, and Xylariales) was the predominant fungal class in the roots of plants from high radioactive environment.

Among the endophytic fungi of $K$. schrenkianum roots, the abundance of ascomycetous classes Leotiomycetes, Sordariomycetes, and unassigned fungi significantly varied among the radiation levels (Table S2). Meanwhile, among the endophytic fungi from aerial tissues, the abundance of basidiomycetous class Agaricomycetes and unassigned fungi significantly varied among the radiation levels. In the whole plant, abundance of the members of classes Leotiomycetes, Sordariomycetes, and Mortierellomycetes and that of the members of phyla Aphelidiomycota and Rozellomycota significantly differed among the radiation levels.

\subsection{Richness of endophytes}

The average taxon richness at species level of endophytic fungi in the whole fungal communities across control 
and three radiation levels was $127.30 \pm 29.21$. Richness of endophytic fungi in aerial tissues was $150.60 \pm$ 12.90 and in roots was $104 \pm 21.01$. Two-way ANOVA indicated that tissue origin significantly influenced the richness of endophytic fungi $(p<0.001)$. T-test showed that the fungal richness was significantly more in aerial tissues than in roots $(\mathrm{df}=31.543, p<0.001)$. In contrast, the bacterial richness was significantly less in aboveground parts than in belowground parts $(\mathrm{df}=30.122, p<0.001)$. The richness of endophytic bacteria across control and three radiation levels was $163.60 \pm 27.95$ in aerial tissues, while it was 252.90 \pm 49.15 in roots. Two-way ANOVA showed that both the tissue types $(p<0.001)$ and its interaction with radiation level $(p=0.029)$ significantly affected the richness of endophytic bacteria.

The richness of endophytic bacteria and fungi in aerial tissues was similar among the control and three radiation levels (Fig. 2). In addition, the richness of endophytic fungi in roots was the same among the different radiation levels while the richness of endophytic bacteria at low radiation level was significantly higher than that in control (Fig. 2).

\subsection{Community composition among treatments}

Current results indicated significant difference in community composition between two tissue types and across control and treatments. NMDS ordination revealed differences in both bacterial and fungal community compositions between aerial tissues and roots (Fig. 3a, b). ANOSIM results showed larger differences in community composition among the groups based on tissue type compared with those based on radiation level for both bacterial and fungal endophytes. We further investigated the community composition of endophytes in different tissues of $K$. schrenkianum. Bacterial communities in roots and fungal communities in both aerial tissues and roots showed significant differences in composition among the radiation levels (Fig. 3df). Meanwhile, the bacterial communities in aerial tissues showed no significant differences in composition among the radiation levels (Fig. 3c). PerMANOVA indicated that the community composition of endophytic bacteria and fungi was significantly affected by tissue type $\left(F_{1,39}=12.021, \mathrm{R}^{2}=0.220, p=0.001 ; F_{1,39}\right.$ $\left.=15.304, \mathrm{R}^{2}=0.242, p=0.001\right)$, radioactive level $\left(F_{3,39}=2.022, \mathrm{R}^{2}=0.111, p=0.001 ; \mathrm{x} F 3,39=3.143\right.$, $\left.\mathrm{R}^{2}=0.149, p=0.001\right)$, and their interaction $\left(F_{3,39}=1.534, \mathrm{R}^{2}=0.084, p=0.032 ; F_{3,39}=2.155, \mathrm{R}^{2}=\right.$ $0.102, p=0.002$ ). PerMANOVA subjected to microbial communities in different tissues separately indicated that the composition was affected by radiation level in case of bacterial communities in roots $\left(F_{3,19}=\right.$ $\left.2.164, \mathrm{R}^{2}=0.289, p=0.002\right)$ and fungal communities in aerial tissues $\left(F_{3,19}=2.189, \mathrm{R}^{2}=0.291, p=\right.$ $0.001)$ and roots $\left(F_{3,19}=2.885, \mathrm{R}^{2}=0.351, p=0.001\right)$.

\subsection{Correlation network}

Correlation network analysis indicated that positive correlation occurred within prokaryotic or eukaryotic groups in microbiota of aerial parts and roots. In aerial tissues, positive correlation was observed among endophytic bacterial genera, while negative correlation occurred among fungal genera (Fig. 4a). For example, unidentified fungal OTU_673 and OTU_702 showed strong negative correlations to several fungal taxa. Meanwhile, positive correlation could be also observed between prokaryotic or eukaryotic members. In roots, bacterial or fungal members in the community were more connected (Fig. 4b). Concatenated negative correlations were observed between prokaryotic and eukaryotic members, which suggested intensive antagonism between bacteria and fungi in roots. These findings indicate denser inter-kingdom interactions in the roots rather than in the aerial tissues.

Network analysis of endophytic bacterial communities suggested a minor effect of radiation on the topology of endophytic bacterial network. The network of endophytic bacterial communities in aerial tissues showed similar connectance and modularity across control and three radioactive treatments (Table 1). Meanwhile, the root bacterial communities showed higher connectance in control and similar modularity in control and radioactive environments. Simultaneously, radiation showed a strong effect on the topology of endophytic fungal correlation network. Higher modularity and lower connectance were observed in all radiation levels compared to control regardless of the tissue type (aerial tissues or roots) (Table 1).

\subsection{Genetic diversity}


Pairwise distances calculated based on "K80" model among all ASVs available in a certain genus were used to evaluate the genetic diversity of microbial population in present study. The results showed that genetic diversity differed within control and different radiation levels for both bacterial and fungal genera. Significant differences were observed in the genetic diversity of 51 bacterial genera and 29 fungal genera (Fig. 5; Table S3 for all bacterial and fungal genera proceeded to one-way ANOVA). Intriguingly, the average genetic distance among more than half of the genera in both bacterial and fungal communities was more in the radioactive environments than that in control (Fig. 5; Table S3 for bacterial genera and Table S4 for fungal genera). Meanwhile, the overall genetic diversity at genus level of both bacterial and fungi in whole plant were higher in the radioactive environments than those in control treatments tested with one-way ANOVA (Table S5).

\subsection{Effects of environmental factors on endophyte abundance or richness}

GLM was used to assess the effect of different environmental factors on endophyte abundance or richness at bacterial genus level or fungal species level. The results showed that richness of bacterial genus negatively correlated with $\mathrm{pH}$ and $\mathrm{TN}$ for the whole plant bacterial communities and positively correlated with roots (Table 2). The bacterial communities in roots showed the same trend with the whole plant bacterial communities, that the richness negatively correlated with $\mathrm{pH}$ and $\mathrm{TN}$. The richness of fungal taxa for the whole plant fungal communities negatively correlated with roots. The fungal communities in roots positively correlated with TN and negatively correlated with OM. There was no significant correlation among bacterial or fungal diversity in aerial tissues and environmental factors.

The abundance of bacterial genera Cupriavidus andBrevibacterium positively correlated with $\mathrm{P}$ and $\mathrm{OM}$ in whole plant bacterial community (Table 3). The abundance of fungal taxaSaitozyma podzolica, Sporormiaceae FOTU_583, AporosporaFOTU_199, fungal genus Saitozyma and Neocamarosporium, and class Dothideomycetes positively correlated with roots. No fungal group showed significant correlation with abiotic environmental factors except Acremonium chrysogenum, which negatively correlated with medium radiation level.

\section{Discussion}

Studies have reported differences in endophytic communities between root and aerial tissues in various plants (Ma, Lv, Warren, \& Gong, 2013; Su, Guo, \& Hyde, 2010). In addition to the difference in biochemical environments between roots and aerial tissues, distinct environmental propagule pools above ground and underground, colonizing and coexisting mechanisms with roots and aerial tissues, and inter-tissue transport limitation contribute to the differences in communities (Fang et al., 2019; Su et al., 2010; X. Sun et al., 2012). Community composition in K. schrenkianum was different between aerial tissues and roots for both bacteria and fungi. Interestingly, the diversity of endophytic bacteria in aerial tissues was less than that in roots, whereas the diversity of endophytic fungi showed an opposite pattern. Microbial species in soil is generally more than that in air. Therefore, the bacterial communities might have largely assembled by immigrating from propagules from the environmental source. However, a differential selection by the tissue significantly shaped the fungal communities in present study. The community composition of endophytic fungi determined by the host is restricted by the environmental propagule pool. Soil related taxa, including Saitozyma podzolica and Sporormiaceae_F_OTU_583, showed significant preference for the root in K. schrenkianum . Researchers first identified $S$.podzolica (previously Candida podzolica) as a soil yeast (Babjeva \& Reshetova, 1975; Liu et al., 2015); Sporormiaceae members are cosmopolitan saprobes (Cannon \& Kirk, 2007). These microbes might be soil inhabitants and be able to colonized $K$.schrenkianum and switched between endophytism and saprophytism (Xiang Sun, Guo, \& Hyde, 2011). In summary, our research suggested that the assembly of both bacterial and fungal endophytic microbiota in roots of K. schrenkianum were affected by soil microbial propagule pools, while roots have stronger selection to fungal colonizer.

In addition to the differences in community composition, our study demonstrates differences between aerial tissues and roots in the responses of endophytic microbiota to environmental factors. The diversity of endophytic microbiota in roots correlated with soil chemical characteristics while the microbiota in aerial tissues showed no response to varied environmental factors. We observed increased diversity in endophytes 
associated with root from control treatment to radioactive environments and not in endophytes associated with aerial tissues. This finding indicates larger effect of environment factors on root endophytic microbiota.

Soil pH is a major determinant of microbial community structure and assembly (Fan et al., 2018; Lauber, Hamady, Knight, \& Fierer, 2009; Rousk et al., 2010). Lauber et al. (2009) stated that soil pH predicted the composition of soil bacterial communities, and phylogenetic diversity attained a peak at near neutral $\mathrm{pH}$. Diversity of endophytic bacteria showed a significant negative correlation with soil $\mathrm{pH}$ of our study sites that ranged from 8.8 to 9.6. This is consistent with the findings of Lauber et al. (2009). Conclusively, the recruitment of root bacterial microbiota is largely dependent on the diversity of soil species. However, we found no correlation between $\mathrm{pH}$ and fungal diversity. Rousk et al. had suggested that fungi prefer a wider $\mathrm{pH}$ range for optimal growth compared with bacteria Rousk et al. (2010).

In a recent study, Bahram et al. (2018) reported strong antagonism between fungi and bacteria globally. In $K$. schrenkianum, we found limited interaction between bacteria and fungi; however, there was bacterial-fungal competition at the niche level. The co-occurrence correlations are prone to restrict to the intra-kingdom members of bacteria and fungi. Meanwhile, inter-kingdom members were negatively correlated. In the roots, bacteria and fungi were less correlated, which indicates less competition in the rhizosphere.

Despite the presence of various chemical factors of soil, radiation was the dominant factor that structured the endophytic communities. Higher genetic diversity was found in radioactive environments for both bacterial and fungal communities. However, mutations due to ionizing radiation and its effect on genetic diversity need to be investigated. Environmental stress resulted in increase in genetic diversity in the community. Nevo (2001) discovered higher genetic diversity in several tested model organisms under stressful environments with thermal, chemical, climatic, and biotic stresses. Increase in genetic diversity enhanced probability of population survival (Lande \& Shannon, 1996), and populations with low genetic diversity had reduced fitness and increased extinction rates (Markert et al., 2010). Therefore, higher genetic diversity acts as an inherent mechanism of community assembly to maintain the community under moderately stressed environments.

Meanwhile, the current study also revealed increased community diversity in roots at radiation stressed treatments apart from the increased population genetic diversity. Diversity as an essential descriptive and metrological feature, could predict the stability, function, and productivity of an ecosystem (Allison \& Martiny, 2008; Delgado-Baquerizo et al., 2020; Tilman, 1996). High species and phylogenetic diversity are related to high functional diversity and redundancy; however, it is difficult to establish direct correspondence yet, because studies that convincingly tested the linkage between phylogeny and physiology in microbial communities are limited (Allison \& Martiny, 2008). High diversity of endophytic communities in K. schrenkianum roots might imply that the radionuclide sediments in soil severely stressed the root niche and consequently diverse microbes assembled to maintain a stable endophytic microbial communities.

Environmental radiation evoked different responses in bacterial and fungal communities. Vries et al. (2018) showed that bacterial networks were less stable under drought stress than fungal networks in grassland mesocosms set in UK. However, in the present study, the community structure of endophytic fungi was sensitive to radiation more than that of endophytic bacteria. Fungal co-occurrence networks were more fragmented under radiation-stressed environments; connectance decreased and modularity increased at all radiation levels. We observed significant differences in the community composition of fungi in both aerial tissues and roots and of bacteria in roots across all treatments. In addition, the fungal community composition showed dramatic differences at class level and at species level across treatments.

Current study is the first to investigate the community structure of plant symbiotic microbiota under radioactive stress. Nevertheless, present study gave additional questions when answering to mechanisms of community assembly and maintenance under the extreme environments. We did not investigate the correlation between ionizing radiation-induced mutation and increase in genetic diversity and the inherent mechanism that drives community assembly under radiation-stressed environment. Besides, we did not analyse the OTUs identified as unassigned fungi, which showed extraordinary abundance and dominance in roots. The OTUs matched (low E-value and identity) the members of phylum Ascomycota (data not shown); however, 
these fungi are still unknown (since no strain was recovered from the plant). Further studies are necessary to expand our knowledge to plant-microbe symbiosis under radioactive environments.

\section{Acknowledgements}

This research work was supported by National Natural Science Foundation of China (31760009), Project of Innovation Talents Cultivation for Young Science in Xinjiang of China (2017Q045), Improvement Project of Agricultural Science and Capacity of Technology Innovation Platform in Xinjiang Academy of Agricultural Sciences (nkypt005), and Special Project for Basic Scientific Activities of Non-profit Institutes Supported the Government of Xinjiang Uyghur Autonomous Region (KY2019021).

\section{Author contributions}

J.Z., X.S., and Z.D.Z. conceived the project design and performed the research. J.Z., Q.Y.T., M.Y.G., L.J.Z. performed sampling and laboratory work. X.S., M.H. performed bioinformatics analysis. J.Z., X.S. wrote the manuscript with significant contributions from A.S. and H.L.Y. All authors read and approved the final manuscript.

\section{ORCID}

Dr. Jing Zhu https://orcid.org/0000-0001-8332-9378

Dr. Xiang Sun https://orcid.org/0000-0002-6875-4971

\section{References}

Allison, S. D., \& Martiny, J. B. H. (2008). Resistance, resilience, and redundancy in microbial communities. Proceedings of the National Academy of Sciences of the United States of America, 105 , 11512-11519. doi:10.1073/pnas.0801925105

Allred, D. M., \& Beck, D. E. (1963). Ecological distribution of some rodents at Nevada atomic test site. Ecology, 44 (1), 211-214. doi:10.2307/1933209

Arnold, A. E., Mejía, L. C., Kyllo, D., Rojas, E. I., Maynard, Z., Robbins, N., \& Herre, E. A. (2003). Fungal endophytes limit pathogen damage in a tropical tree. Proceedings of the National Academy of Sciences of the United States of America, 100 (26), 15649-15654. doi:10.1073/pnas.2533483100

Auguie, B. (2017). gridExtra: Miscellaneous Functions for "Grid" Graphics. Retrieved from https://CRAN.R-project.org/package=gridExtra

Babjeva, I. P., \& Reshetova, I. S. (1975). New yeast species from soil - candida podzolica sp n. Mikrobiologiia, 44 (2), 333-338

Bahram, M., Hildebrand, F., Forslund, S. K., Anderson, J. L., Soudzilovskaia, N. A., Bodegom, P. M., . . . Bork, P. (2018). Structure and function of the global topsoil microbiome. Nature, 560 , 233-237. doi:10.1038/s41586-018-0386-6

Beatley, J. C. (1964). Vascular flora of Nevada Test Site Nye county Nevada. American Journal of Botany, 51 (6P2), 687

Benjamini, Y., \& Hochberg, Y. (1995). Controlling the false discovery rate: a practical and powerful approach to multiple testing. Journal of the Royal Statistical Society Series B-Methodological, 57 (1), 289-300

Bodenhausen, N., Horton, M. W., \& Bergelson, J. (2013). Bacterial communities associated with the leaves and the roots of Arabidopsis thaliana . Plos One, 8 (2), e56329. doi:10.1371/journal.pone.0056329

Callahan, B. J., Sankaran, K., Fukuyama, J. A., McMurdie, P. J., \& Holmesa, S. P. (2016). Bioconductor workflow for microbiome data analysis: From raw reads to community analyses. . F1000Research, 5, 1492. doi:10.12688/f1000research.8986.2

Cannon, P., \& Kirk, P. (2007). Fungal Families of the World . Wallingford, UK: CABI 
Chelius, M. K., \& Triplett, E. W. (2001). The diversity of archaea and bacteria in association with the roots of Zea mays L.Microbial Ecology, 41 , 252-263. doi:10.1007/s002480000087

Compant, S., Heijden, M. G. A. V. D., \& Sessitsch, A. (2010). Climate change effects on beneficial plant-microorganism interactionsFEMS Microbiology Ecology, 73 (2), 197-214. doi:10.1111/j.15746941.2010.00900.x

Csardi, G., \& Nepusz, T. (2006). The igraph software package for complex network research. InterJournal, Complex Systems , 1695

Delgado-Baquerizo, M., Reich, P. B., Trivedi, C., Eldridge, D. J., Abades, S., Alfaro, F. D., . . Singh, B. K. (2020). Multiple elements of soil biodiversity drive ecosystem functions across biomes. Nature Ecology $\mathscr{E}$ Evolution, 4 , 210-220. doi:10.1038/s41559-019-1084-y

Dormann, C. F., Fründ, J., Blüthgen, N., \& Gruber, B. (2009). Indices, graphs and null models: Analyzing bipartite ecological networks. The Open Ecology Journal, 2 , 7-24. doi:10.2174/1874213000902010007

Durrell, L. W., \& Shields, L. M. (1960). Fungi isolated in culture from soils of the Nevada Test Site. Mycologia, 52 (4), 636-641. doi:10.2307/3756096

Faith, D. P., Minchin, P. R., \& Belbin, L. (1987). Compositional dissimilarity as a robust measure of ecological distance Vegetatio, 69 , 57-68

Fan, K., Weisenhorn, P., Gilbert, J. A., Shi, Y., Bai, Y., \& Chu, H. (2018). Soil pH correlates with the co-occurrence and assemblage process of diazotrophic communities in rhizosphere and bulk soils of wheat fields. Soil Biology and Biochemistry, 121 , 185-192. doi:10.1016/j.soilbio.2018.03.017

Fang, K., Miao, Y.-F., Chen, L., Zhou, J., Yang, Z.-P., Dong, X.-F., \& Zhang, H.-B. (2019). Tissue-specific and geographical variation in endophytic fungi of Ageratina adenophora and fungal associations with the environment. Frontiers in Microbiology, 10 , 2919. doi:10.3389/fmicb.2019.02919

Gange, A. C., Eschen, R., Wearn, J. A., Thawer, A., \& Sutton, B. C. (2012). Differential effects of foliar endophytic fungi on insect herbivores attacking a herbaceous plant. Oecologia, 168 , 1023-1031. doi:10.1007/s00442-011-2151-5

Gardes, M., \& Bruns, T. D. (1993). ITS primers with enhanced specificity for basidiomycetes - application to the identification of mycorrhizae and rusts. Molecular Ecology, 2 (2), 113-118. doi:10.1111/j.1365294X.1993.tb00005.x

Guo, L. D., Hyde, K. D., \& Liew, E. C. Y. (2000). Identification of endophytic fungi from Livistona chinensis based on morphology and rDNA sequences. New Phytologist, 147, 617-630

Lande, R., \& Shannon, S. (1996). The role of genetic variation in adaptation and population persistence in a changing environmentEvolution, 50 (1), 434-437. doi:10.2307/2410812

Lauber, C. L., Hamady, M., Knight, R., \& Fierer, N. (2009). Pyrosequencing-based assessment of soil pH as a predictor of soil bacterial community structure at the continental scale. Applied and Environmental Microbiology, 75 (15), 5111-5120. doi:10.1128/AEM.00335-09

Lavrinienko, A., Tukalenko, E., Mappes, T., \& Watts, P. C. (2018). Skin and gut microbiomes of a wild mammal respond to different environmental cues. Microbiome, 6 , 209. doi:10.1186/s40168-018-0595-0

Liu, X. Z., Wang, Q. M., Goeker, M., Groenewald, M., Kachalkin, A. V., Lumbsch, H. T., . . Bai, F. Y. (2015). Towards an integrated phylogenetic classification of the Tremellomycetes. Studies in Mycology, 81 , 85-147. doi:10.1016/j.simyco.2015.12.001

Ma, B., Lv, X. F., Warren, A., \& Gong, J. (2013). Shifts in diversity and community structure of endophytic bacteria and archaea across root, stem and leaf tissues in the common reed, Phragmites australis, along 
a salinity gradient in a marine tidal wetland of northern China.Antonie Van Leeuwenhoek International Journal of General and Molecular Microbiology, 104 (5), 759-768. doi:10.1007/s10482-013-9984-3

Markert, J. A., Champlin, D. M., Gutjahr-Gobell, R., Grear, J. S., Kuhn, A., Jr, T. J. M., . . . Nacci, D. E. (2010). Population genetic diversity and fitness in multiple environments. Bmc Evolutionary Biology, 10 , 205. doi:10.1186/1471-2148-10-205

Mendiburu, F. d. (2019). agricolae: Statistical Procedures for Agricultural Research. R package version 1.3-1. Retrieved from https://CRAN.R-project.org/package=agricolae

Murrell, P. (2005). R Graphics . London, UK: Chapman \& Hall/CRC Press

Nevo, E. (2001). Evolution of genome-phenome diversity under environmental stress. Proceedings of the National Academy of Sciences of the United States of America, 98 (11),6233-6240. doi:10.1073/pnas.101109298

Nilsson, R., Larsson, K.-H., Taylor, A., Bengtsson-Palme, J., Jeppesen, T., Schigel, D., . . Abarenkov, K. (2019). The UNITE database for molecular identification of fungi: handling dark taxa and parallel taxonomic classifications. Nucleic Acids Research, 47 (D1), D259-D264. doi:10.1093/nar/gky1022

Oksanen, J., Blanchet, F. G., Kindt, R., Legendre, P., Minchin, P. R., O’Hara, R. B., . . Wagner, H. (2016). Package 'vegan': Community ecology package. Retrieved from https://cran.r-project.org

Paradis, E., \& Schliep, K. (2018). ape 5.0: an environment for modern phylogenetics and evolutionary analyses in R Bioinformatics, 35 (3), 526-528. doi:10.1093/bioinformatics/bty633

Porras-Alfaro, A., \& Bayman, P. (2011). Hidden fungi, emergent properties: Endophytes and microbiomes. Annual Review of Phytopathology, 49 , 291-315. doi:10.1146/annurev-phyto-080508-081831

Quast, C., Pruesse, E., Yilmaz, P., Gerken, J., Schweer, T., Yarza, P., . . Glöckner, F. O. (2013). The SILVA ribosomal RNA gene database project: improved data processing and web-based tools. Nucleic Acids Research, 41 (D1), D590-D596. doi:10.1093/nar/gks1219

R Development Core Team. (2016). R: A language and environment for statistical computing. Retrieved from http://www.R-project.org

Rickard, W. H., \& Beatley, J. C. (1965). Canopy-coverage of the desert shrub vegetation mosaic of the Nevada Test Site. Ecology, 46 (4), 524-529. doi:10.2307/1934886

Rodriguez, R. J., Henson, J., Volkenburgh, E. V., Hoy, M., Wright, L., Beckwith, F., . . . Redman, R. S. (2008). Stress tolerance in plants via habitat-adapted symbiosis. Isme Journal, 2 , 404-416. doi:10.1038/ismej.2007.106

Rousk, J., Bååth, E., Brookes, P. C., Lauber, C. L., Lozupone, C., Caporaso, J. G., . . Fierer, N. (2010). Soil bacterial and fungal communities across a $\mathrm{pH}$ gradient in an arable soil. The ISME Journal, 4, 1340-1351. doi:10.1038/ismej.2010.58

Saikkonen, K., Faeth, S. H., Helander, M., \& Sullivan, T. J. (1998). Fungal endophytes: A continuum of interactions with host plants.Annual Review of Ecology and Systematics, 29 , 319-343. doi:doi.org/10.1146/annurev.ecolsys.29.1.319

Scrucca, L. (2004). qcc: an R package for quality control charting and statistical process control. $R$ News, 4 (1), 11-17

Shields, L. M., \& Drouet, F. (1962). Distribution of terrestrial algae within Nevada Test Site. American Journal of Botany, 49 (6), 547-554. doi:10.2307/2439709

Shields, L. M., Durrell, L. W., \& Sparrow, A. H. (1961). Preliminary-observations on radiosensitivity of algae and fungi from soils of Nevada Test Site. Ecology, 42 (2), 440-441. doi:10.2307/1932103 
Su, Y. Y., Guo, L. D., \& Hyde, K. D. (2010). Response of endophytic fungi of Stipa grandis to experimental plant function group removal in Inner Mongolia steppe, China Fungal Diversity, 43 (1), 93-101. doi:10.1007/s13225-010-0040-6

Sun, X., Ding, Q., Hyde, K. D., \& Guo, L. D. (2012). Community structure and preference of endophytic fungi of three woody plants in a mixed forest. Fungal Ecology, 5 (5), 624-632. doi:10.1016/j.funeco.2012.04.001

Sun, X., Guo, L. D., \& Hyde, K. D. (2011). Community composition of endophytic fungi in Acer truncatum and their role in decomposition. Fungal Diversity, 47 (1), 85-95. doi:10.1007/s13225-010-0086-5

Tilman, D. (1996). Biodiversity: Population versus ecosystem stability. Ecology, 77 (2), 350-363. doi: $10.2307 / 2265614$

U'Ren, J. M., Lutzoni, F., Miadlikowska, J., Laetsch, A. D., \& Arnold, A. E. (2012). Host and geographic structure of endophytic and endolichenic fungi at a continental scale. American Journal of Botany, 99 (5), 898-914. doi:10.3732/ajb.1100459

Veach, A. M., Morris, R., Yip, D. Z., Yang, Z. K., Engle, N. L., Cregger, M. A., . . Schadt, C. W. (2019). Rhizosphere microbiomes diverge among Populus trichocarpa plant-host genotypes and chemotypes, but it depends on soil origin. Microbiome, 7 , 76. doi:10.1186/s40168-019-0668-8

Venables, W. N., \& Ripley, B. D. (2002). Modern Applied Statistics with S (Fourth ed.). New York: Springer

Vries, F. T. D., Griffiths, R. I., Bailey, M., Craig, H., Girlanda, M., Gweon, H. S., . . . Bardgett, R. D. (2018). Soil bacterial networks are less stable under drought than fungal networks. Nature Communications, 9 , 3033. doi:10.1038/s41467-018-05516-7

Wehrden, H. V., Fischer, J., Brandt, P., Wagner, V., Kümmerer, K., Kuemmerle, T., . . Hostert, P. (2012). Consequences of nuclear accidents for biodiversity and ecosystem services. Conservation Letters, 5 (2), 81-89. doi:10.1111/j.1755-263X.2011.00217.x

White, T., Bruns, T., Lee, S., \& Taylor, J. (1990). Amplification and direct sequencing of fungal ribosomal RNA genes for phylogeneics. In M. Innis, D. Gelfand, J. Sninsky, \& T. White (Eds.), PCR protocols: a guide to methods and applications (pp. 315-322). San Diego: Academic

Wickham, H. (2016). ggplot2: Elegant Graphics for Data Analysis : Springer-Verlag New York.

Wilkinson, S. (2018). kmer: an R package for fast alignment-free clustering of biological sequences. R package version 1.0.0. Retrieved from https://cran.r-project.org/package $=$ kmer

Yao, X., Chen, Z., Wei, X., Chen, S., White, J., Huang, X., . . Nan, Z. (2020). A toxic grass Achnatherum inebrians serves as a diversity refuge for the soil fungal community in rangelands of northern China. Plant and Soil, 448 , 425-438. doi:doi.org/10.1007/s11104-020-04440-4

Zhdanova, N. N., Zakharchenko, V. A., \& Haselwandter, K. (2005). Radionuclides and fungal communities. In J. Dighton, J. F. White, \& P. Oudemans (Eds.), The Fungal Community - Its Organization and Role in the Ecosystem (Third Edition ed., pp. 759-768). Boca Raton, FL: CRC Press

Zhdanova, N. N., Zakharchenko, V. A., Vember, V. V., \& Nakonechnaya, L. T. (2000). Fungi from Chernobyl: mycobiota of the inner regions of the containment structures of the damaged nuclear reactor. Mycological Research, 104 (12), 1421-1426. doi:10.1017/S0953756200002756

\section{Hosted file}

title page.pdf available at https://authorea.com/users/377448/articles/494122-effect-ofionizing-radiation-on-the-bacterial-and-fungal-endophytes-of-the-halophytic-plantkalidium-schrenkianum 

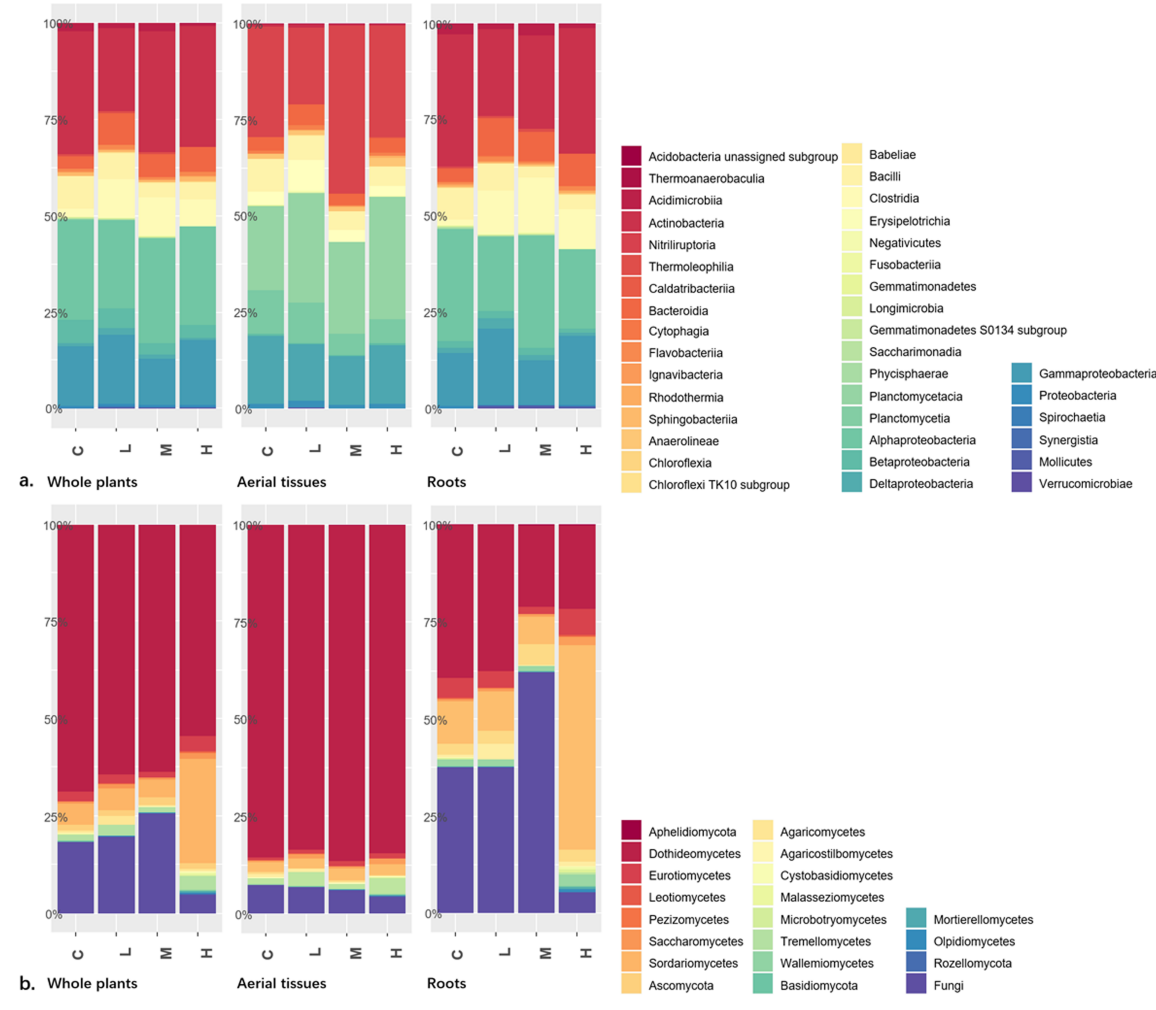


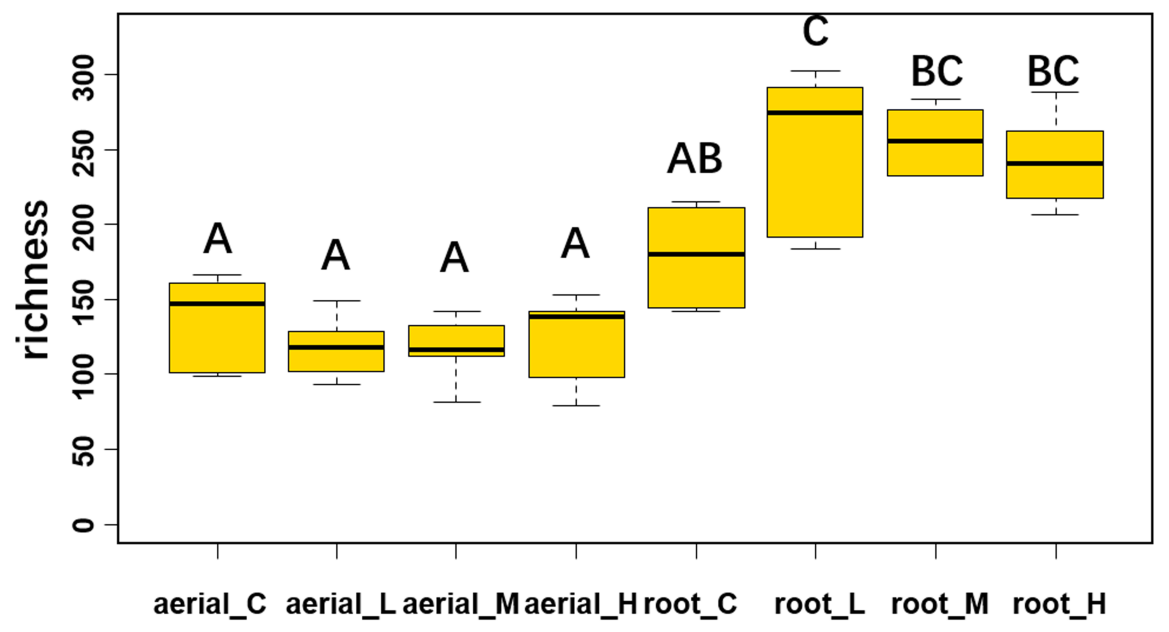

a. Endophytic bacteria

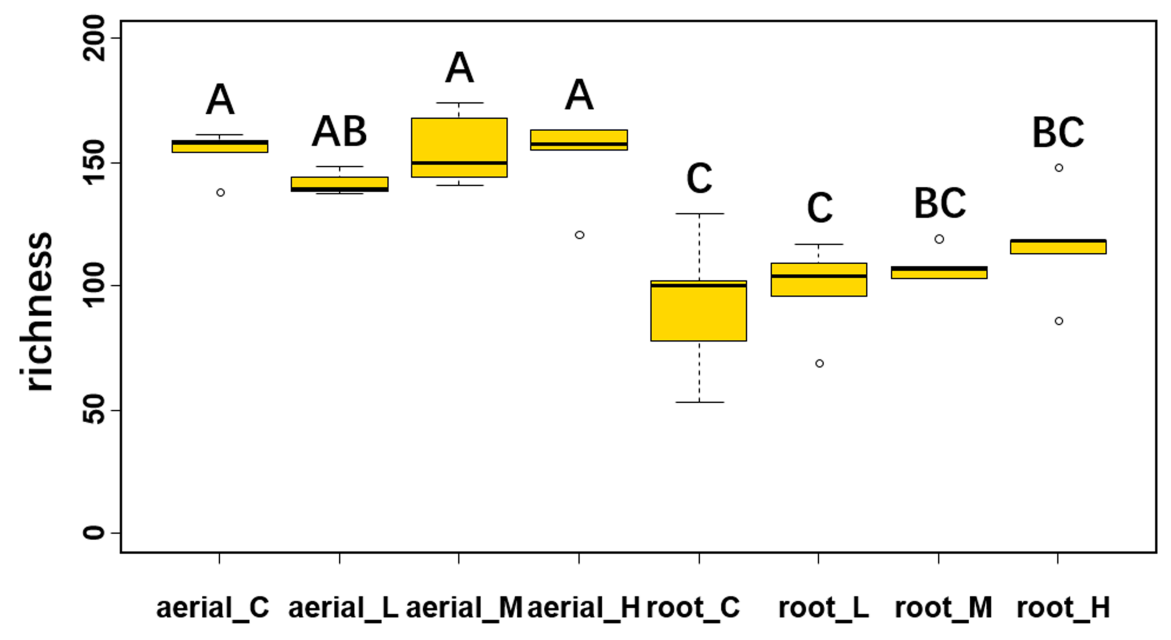

b. Endophytic fungi 

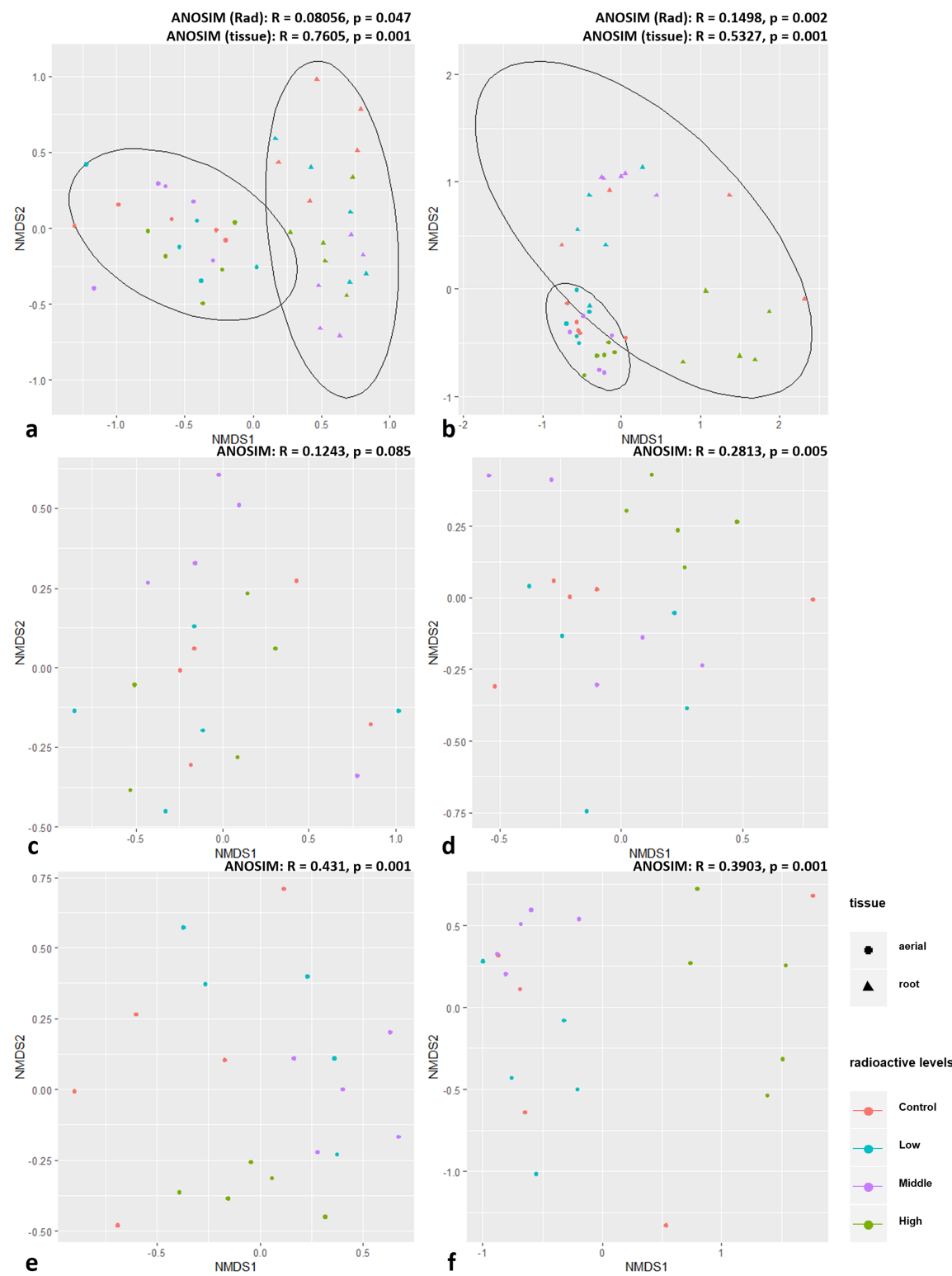

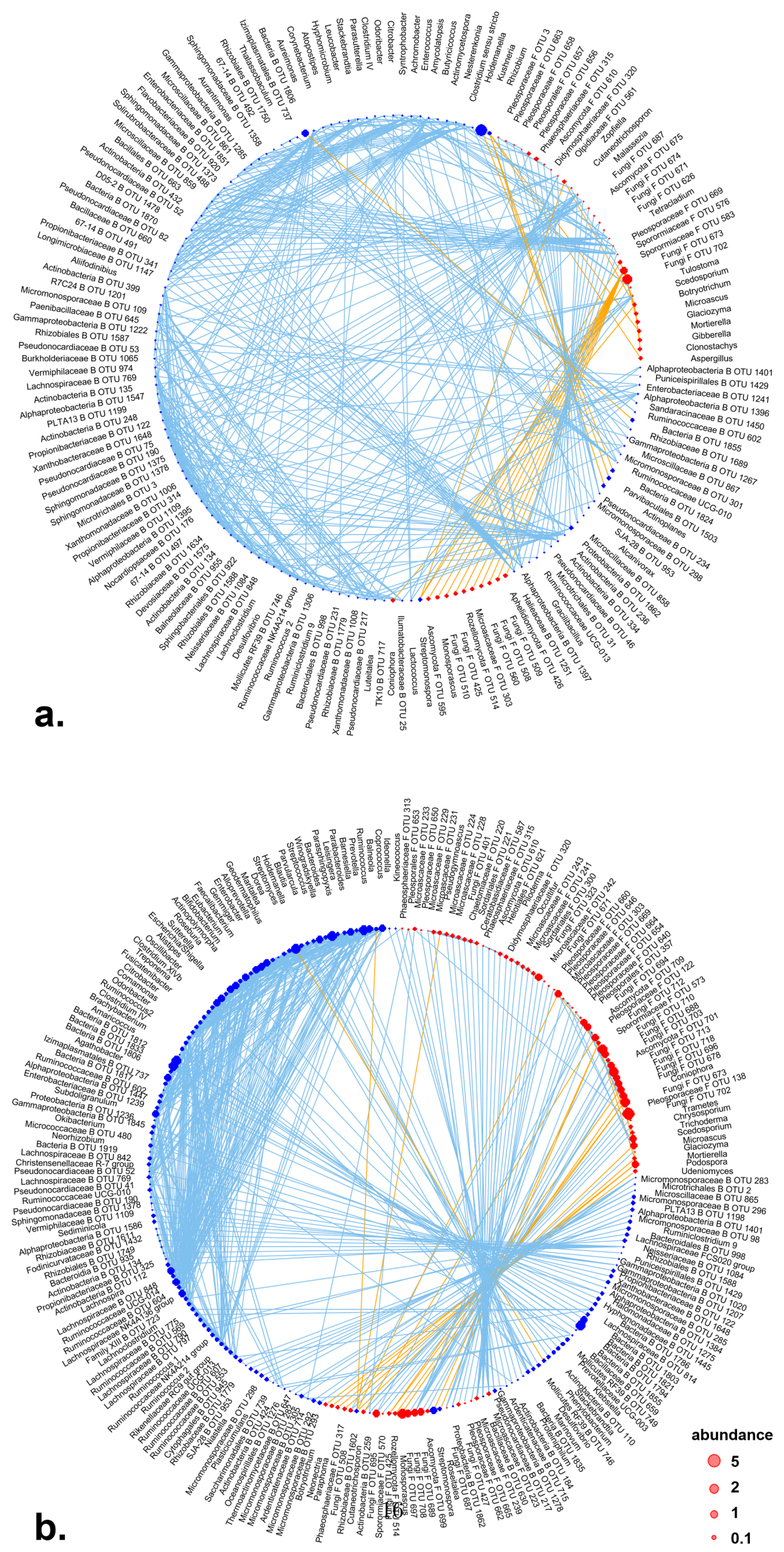


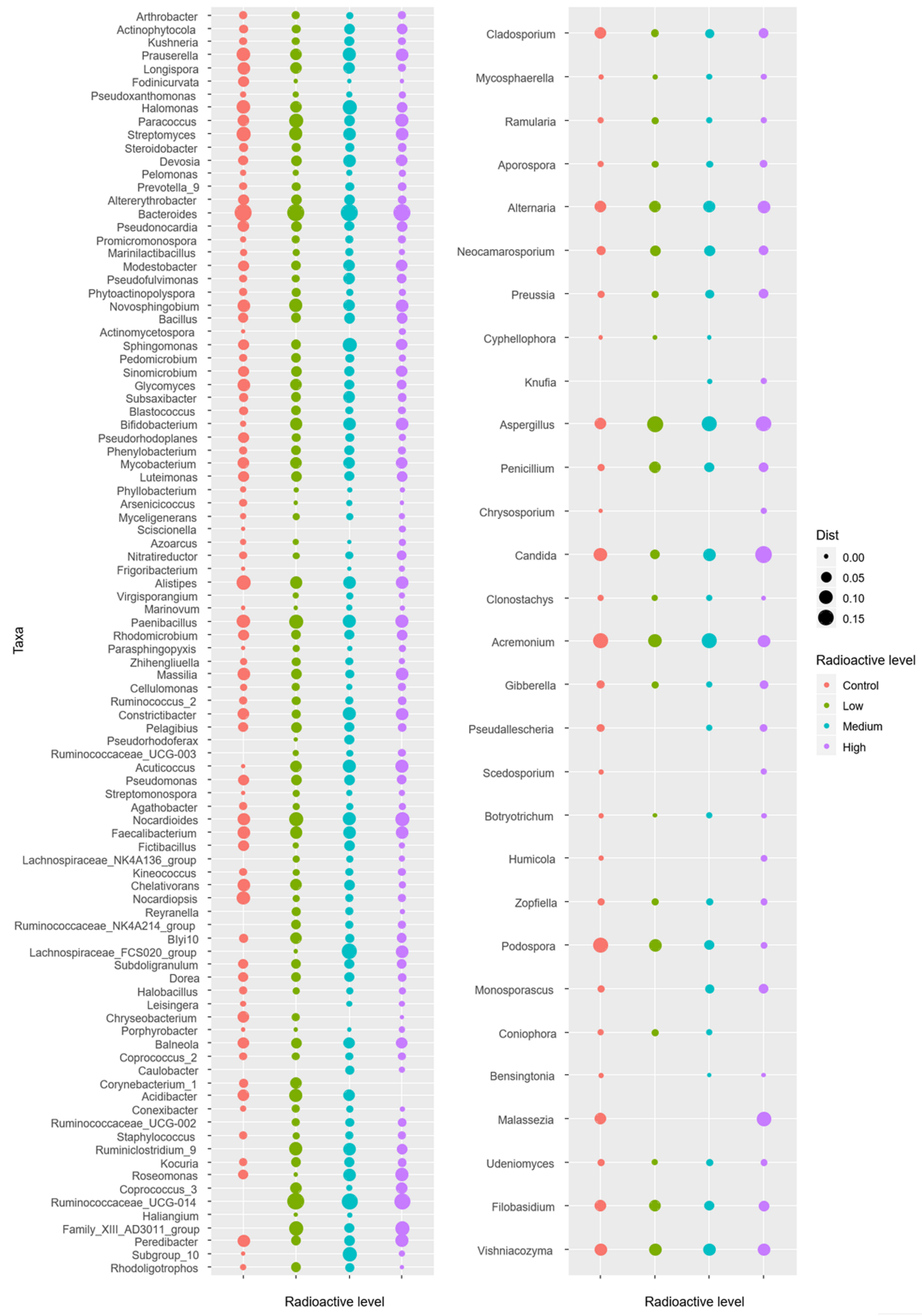

\section{Hosted file}

Table 1.pdf available at https://authorea.com/users/377448/articles/494122-effect-ofionizing-radiation-on-the-bacterial-and-fungal-endophytes-of-the-halophytic-plantkalidium-schrenkianum

\section{Hosted file}

Table 2.pdf available at https://authorea.com/users/377448/articles/494122-effect-ofionizing-radiation-on-the-bacterial-and-fungal-endophytes-of-the-halophytic-plantkalidium-schrenkianum

\section{Hosted file}


Table 3.pdf available at https://authorea.com/users/377448/articles/494122-effect-ofionizing-radiation-on-the-bacterial-and-fungal-endophytes-of-the-halophytic-plantkalidium-schrenkianum 